\title{
Preface: Probiotics and Immunity
}

The human gut is hosted by microbial communities or microbiota that consist of approximately $10^{14}$ bacteria that evolve to establish a symbiotic relationship with the human host in the regulation of physiological homeostasis. The functions of microbiota are numerous and diverse and include the synthesis of amino acids and vitamins, energy extraction from nonabsorbable nutrients, and action against pathogens. In addition, and most importantly, this special symbiotic relationship is critical in its participation of the initiation of both innate and adaptive immunities against foreign pathogens.

Several reports and reviews have been published regarding the important role of the microbiota in maintaining the balance between a healthy environment and a diseased environment. Changes in the composition of gut microbiota have been reported to be associated with several clinical conditions including obesity, metabolic diseases, autoimmune diseases and anergy, acute and chronic intestinal inflammation, uncontrolled bowel syndrome, allergic gastroenteritis, and necrotizing enterocolitis. Modulations of gut microbiota with probiotics have been suggested to be treatments/preventions for different disorders. Probiotics have been defined as viable microbial species that can be ingested for the purpose of altering the gastrointestinal flora in a manner that can provide health benefits.

This special section's content is very restricted and primarily illustrates a few examples of probiotics and immunity. The various chapters list several reviews in the literature on the same subject. Underwood's Probiotics and Innate and Adaptive Immune Responses in Premature Infants reviews the findings that show that some premature infants experience necrotizing enterocolitis and sepsis that can lead to increased risk of morbidity and mortality. Consumption of probiotics by premature infants reduces the risk of necrotizing enterocolitis and death. Although these findings are encouraging, the mechanisms involved are not known. Dr. Underwood reviews the variety of probiotic species that have been cited for their clinical benefits to premature infants, and it appears that probiotics alter intestinal inflammation dysmobility and permeability and introduce different gut microbes for better use of nutrients. He also discusses the role of probiotics in the regulation of both innate and adaptive immune responses.

Goyal and colleagues' Probiotics in Human Health reviews the reported beneficial effects of probiotics in human health based on the composition of the bacterial species. They discuss the benefits of probiotics in the preparation of vaccines because they improve both humoral and cellular immunities. These authors also review the adjuvanticity of probiotics in the prevention and treatment of various diseases. This chapter is an overview updating advances that have been reported regarding the beneficial applications of probiotics in health.

Touchefeu et al.'s Gut Microbiota: Potential Impact on Chemotherapy-Related Adverse and Therapeutic Effects reviews the role of gut microbiota on the maintenance of intestinal integrity and how treatment with chemotherapy results in changes in both composition and gene functions of gut microbiota. Consequently, these changes result in gastrointestinal mucositis. These researchers review the reported literature on preventive measures in cancer and treatment-induced mucositis and discuss how gut microbiota can be beneficial in its partnership with chemotherapy for improving outcome.

Aziz and Bonavida's Activation of Natural Killer Cells by Probiotics reviews the literature on the role of probiotics in the regulation of innate immunity. Several different probiotics are currently being used to affect gut-induced immunity differently. Natural killer (NK) cells in the gut and their role in innate and adaptive immunity have been scarcely investigated. This review reports on the activation of gutassociated NK cells by probiotics, their activation to secrete various cytokines, and how these can affect several diseases. The role of NK cells in health is primordial for fighting infection and cancer, and their activation by probiotics may improve the immune response against such manifestations.

The editor wishes to acknowledge the assistance 
of Leah Moyal, Kevin Li, and Ailina Lao for their diligent editing and preparation of the various articles in this issue.

\section{Guest Editor:}

\section{Benjamin Bonavida}

University of California, Los Angeles

Los Angeles, CA 